\title{
Coronary artery aneurysms and Kawasaki's disease in an adult
}

\author{
D B G OLIVEIRA, R A FOALE, J BENSAID \\ From the Ealing Hospital and Royal Postgraduate Medical School, Hammersmith Hospital, London; and the \\ University Hospital of Limoges, France
}

SUMMARY A 28 year old man had an acute myocardial infarction and was found to have coronary artery aneurysms. These may have been caused by a previous episode of Kawasaki's disease, an entity that should be considered in adults presenting with proximal discrete coronary artery aneurysms.

Since the first major review of coronary artery aneurysms in $1908^{1}$ several other series have been published, the most recent being that of the Coronary Artery Surgery Study which included a total of 978 patients, ${ }^{2}$ although in most of their cases the dilatations represented coronary ectasia.

Atherosclerosis has been considered to be the most importan: cause, and the Coronary Artery Surgery Study's report concluded that aneurysmal coronary disease appeared to be a variant of occlusive coronary atherosclerosis. ${ }^{2}$ Recently, it has, however, become apparent that coronary artery aneurysm is an important complication of Kawasaki's disease (mucocutaneous lymph node syndrome). ${ }^{3}$ We report a case in a young man with minimal risk factors for atherosclerosis who presented with myocardial infarction and who was found at angiography to have coronary artery aneurysms with only minor disease in the rest of his coronary arterial tree.

\section{Case report}

A 28 year old Mauritian-born Asian man developed severe retrosternal chest pain while on holiday in France in November 1982 and was admitted to the intensive care unit of the University Hospital, Limoges. There was no notable past medical history and no previous episodes of chest pain. He could not recall any unusual or severe childhood illnesses. $\mathrm{He}$ was a non-smoker and there was no family history of ischaemic heart disease.

Examination on admission was unremarkable with a blood pressure of $140 / 80 \mathrm{mmHg}$. The electrocar- diogram showed an extensive anterior myocardial infarction with right bundle branch block, and plasma creatine kinase activity was raised at $1260 \mathrm{U} / \mathrm{l}$ (normal $<70 \mathrm{U} / \mathrm{l}$ ). The only other abnormal finding was a raised blood sugar concentration of $20 \mathrm{mmol} / \mathrm{l}(3.6$ $\mathrm{g} / \mathrm{l}$ ).

Apart from an initial persistent tachycardia and gallop rhythm he made an uncomplicated recovery, and the hyperglycaemia was controlled with an insulin infusion. Cardiac catheterisation with angiography was performed 11 days after admission to evaluate his coronary artery tree. Left ventriculography showed a large apical ventricular aneurysm with a mural thrombus. Selective left coronary arteriography showed a large aneurysm in the left main stem and proximal part of the circumflex artery. The left anterior descending artery was occluded near the aneurysm by an apparent thrombus. The distal vessel filled in a retrograde fashion from the right coronary system. There were mild ectatic changes and minor plaques in the remaining coronary vessels (Fig. 1).

He was transferred to Ealing Hospital about three weeks after presentation. Further investigations performed at this stage showed that the results of tests for hepatitis B surface antigen, the Treponema pallidum haemagglutination assay, the Venereal Disease Research Laboratory test, and an autoimmune assay (parietal cell, pancreatic islets, anti-nuclear, smooth muscle, and mitochondria) were all negative. Serum lipid concentrations were: cholesterol $5.2 \mathrm{mmol} / \mathrm{l}(2.0$ $\mathrm{g} / \mathrm{l})$ (normal $3.5-6.5 \mathrm{mmol} / \mathrm{l}(1.3-2.5 \mathrm{~g} / \mathrm{l})$ ), triglycerides $1.4 \mathrm{mmol} / \mathrm{l}(1.2 \mathrm{~g} / \mathrm{l}$ ) (normal $0.9-1.8 \mathrm{mmol} / \mathrm{l}$ $(0.7-1.5 \mathrm{~g} / 1)$ ); blood glucose concentration was 11.1 $\mathrm{mmol} / \mathrm{l}(2.0 \mathrm{~g} / \mathrm{l})$. Cross sectional echocardiography was performed using a phased array $3.5 \mathrm{MHz}$ trans- 


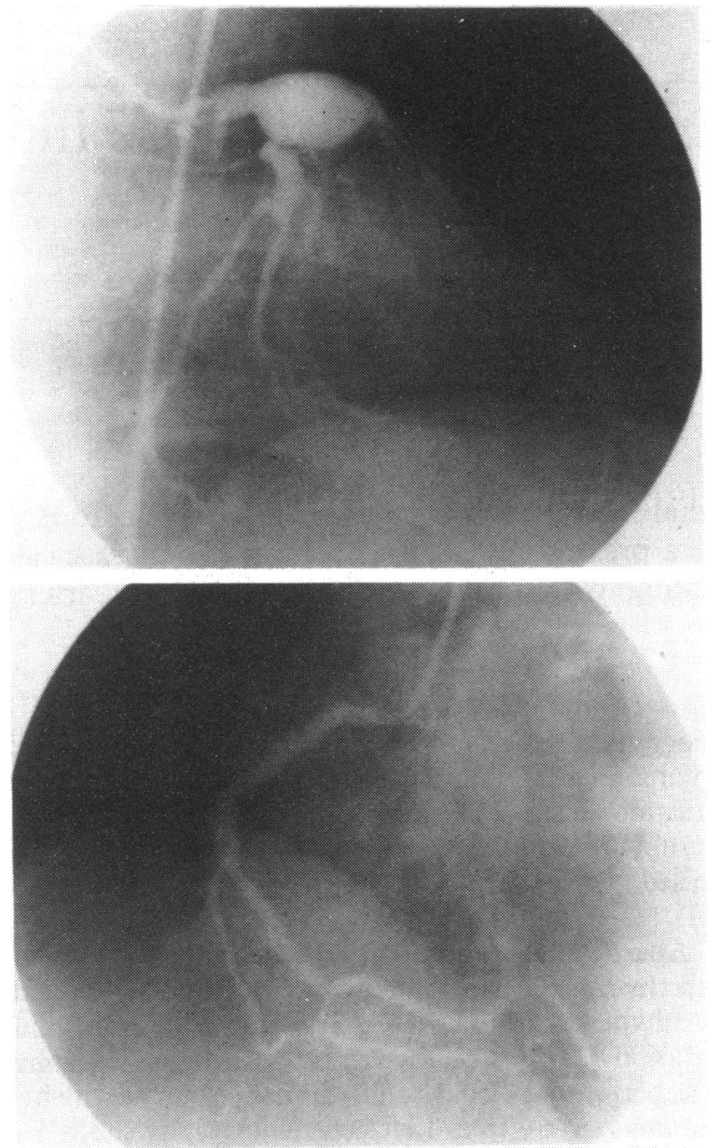

Fig. 1 Coronary arteriogram showing anewrysm affecting the left main stem and proximal circumflex artery, occluded left anterior descending artery (upper panel), and mild ectatic changes in the right coromary artery (lower panel).

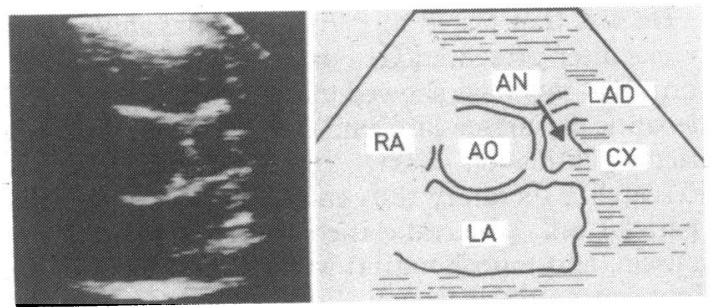

Fig. 2 Cross sectional echocardiogram of the parastermal shiort axis view at the level of the aortic root showing the aneurysm $(A N)$ as a constant space in the region of the left main coromary artery that gives origin to the left anterior descending (LAD) and circumflex $(C X)$ vessels. $R A$; right atrium; $A O$, aorta; $L A$, left atrium. ducer. The left coronary artery aneurysm was well visualised and arose $1 \mathrm{~cm}$ from the origin of the left main stem and extended to the proximal circumflex and left anterior descending branches. The aneurysm measured $1.5 \times 1.3 \mathrm{~cm}$ in its maximum and minimum dimensions (Fig. 2). On examination of the left ventricle there was pronounced anteroseptal dyskinesia, although no thrombus was visualised within the cavity.

His present treatment consists of anticoagulation with warfarin and control of his diabetes with diet and glibenclamide; he remains asymptomatic on follow up at eight months.

\section{Discussion}

We propose that the probable sequence of events in this case was thrombosis within the aneurysm followed by distal embolisation. Thus a myocardial infarct evolved with the subsequent development of a left ventricular aneurysm. Anticoagulation treatment seemed, therefore, to be reasonable, and additional support for this approach is found in the mural thrombus visualised at angiography.

The aetiology of the coronary artery aneurysms cannot be determined with certainty. Trauma, mycotic/embolic, and syphilis would seem to be eliminated as causes by the history and negative serological findings. Apart from mild diabetes, there were no other risk factors for atherosclerosis. In addition, when aneurysms are associated with atherosclerosis the overwhelming majority of cases have pronounced coronary artery stenoses ${ }^{2}$; in our patient the other coronary arteries showed mild ectasia only. Although a congenital origin cannot be discounted, well documented reports of true congenital aneurysms not associated with arteriovenous fistula and present since birth are rare and often occur in the presence of other congenital abnormalities. ${ }^{4}$ Polyarteritis nodosa as a cause of coronary artery aneurysms seems to have a predilection for infancy and childhood, and many of the cases reported bear a notable clinical resemblance to Kawasaki's disease ${ }^{5}$; furthermore, the pathological findings in the two conditions are identical. 67 As most of the descriptions of juvenile polyarteritis with coronary artery aneurysms appeared before Kawasaki's original English language report ${ }^{6}$ and widespread recognition of the latter entity there may be considerable overlap between the two conditions. ${ }^{7}$ Coronary artery aneurysm appears to be a relatively frequent complication, occurring in one study in seven out of 20 apparently unselected survivors undergoing coronary arteriography. ${ }^{3}$ These aneurysms may persist: one patient was aged 26 years, having had a presumed episode of Kawasaki's disease at the age of 14 years. ${ }^{8}$ Although there was no history suggesting Kawasaki's 
disease in our patient, most of the features of the disease are non-specific, and the more distinctive manifestations such as the rash followed by desquamation do not occur in all cases. ${ }^{6}$ This aetiology is partly supported by the appearance of the aneurysms, which are not typical of those occurring in atherosclerosis 9 but bear a notable resemblance to some of the lesions found in Kawasaki's disease ${ }^{3810}$ which often seem to affect the left main coronary artery. Thus, as well as being a well recognised association in childhood, Kawasaki's disease should also be considered in the adult presenting with coronary artery aneurysms.

Further management may involve surgery or medical treatment alone. Surgery has been recommended for all but minor aneurysms in view of the risk of thrombosis or rupture, ${ }^{11}$ and there have also been reports of surgery for aneurysms secondary to Kawasaki's disease. 8 The data from the Coronary Artery Surgery Study, however, show that the prognosis in cases secondary to atherosclerosis is that of the underlying obstructive disease, with no extra risk being conferred by the presence of aneurysms. ${ }^{2}$ Although no comparable data exist for Kawasaki's disease, we consider that surgery was not justified in this asymptomatic man with only minor disease in his other coronary arteries. His treatment at present, therefore, consists of anticoagulation only. We plan to monitor the appearances of the coronary aneurysm with cross sectional echocardiography. The use of this technique in Kawasaki's disease has been reported ${ }^{10}$ and may be a useful non-invasive method of assessing and following the progress of coronary artery aneurysms in this condition.

\section{References}

1 Auffermann H. Aneurysmata der herzarterien. Berlin: LM
Barschall, 1908

2 Swaye PS, Fisher LD, Litwin P, et al. Aneurysmal coronary artery disease. Circulation 1983; 67: 134-8.

3 Kato H, Koike S, Yamamoto M, Ito Y, Yano E. Coronary aneurysms in infants and young children with acute febrile mucocutaneous lymph node syndrome. $\mathcal{F}$ Pediatr 1975; 86: 892-8.

4 Crocker DW, Sobin S, Thomas WC. Aneurysms of the coronary arteries. Am f Pathol 1957; 33: 819-43.

5 Roberts FB, Fetterman GH. Polyarteritis nodosa in infancy. $\mathcal{F}$ Pediatr 1963; 63: 519-29.

6 Kawasaki T, Kosaki F, Okawa S, Shigematsu I, Yanagawa $\mathrm{H}$. A new infantile acute febrile mucocutaneous lymph node syndrome (MLNS) prevailing in Japan. Pediatrics 1974; 54: 271-6.

7 Landing BH, Larson EJ. Are infantile periarteritis nodosa with coronary artery involvement and fatal mucocutaneous lymph node syndrome the same? Comparison of 20 patients from North America with patients from Hawaii and Japan. Pediatrics 1979; 59: 651-62.

8 Kitamura S, Kawachi K, Harima R, Sakakibara T, Hirose H, Kawashima Y. Surgery for coronary heart disease due to mucocutaneous lymph node syndrome (Kawasaki's disease). Am f Cardiol 1983; 51: 444-8.

9 Swanton RH, Thomas ML, Coltart DJ, Jenkins BS, Webb-Peploe MM, Williams BT. Coronary artery ectasia-a variant of occlusive coronary arteriosclerosis. Br Heart f 1978; 40: 393-400.

10 Yoshikawa J, Yanagihara K, Owaki T, et al. Crosssectional echocardiographic diagnosis of coronary artery aneurysms in patients with the mucocutaneous lymph node syndrome. Circulation 1979; 59: 133-9.

11 Glickel SZ, Maggs PR, Ellis FH Jr. Coronary artery aneurysm. Ann Thorac Surg 1978; 25: 372-6.

Requests for reprints to Dr D B G Oliveira, Renal Unit, Hammersmith Hospital, Du Cane Road, London W12 0HS. 